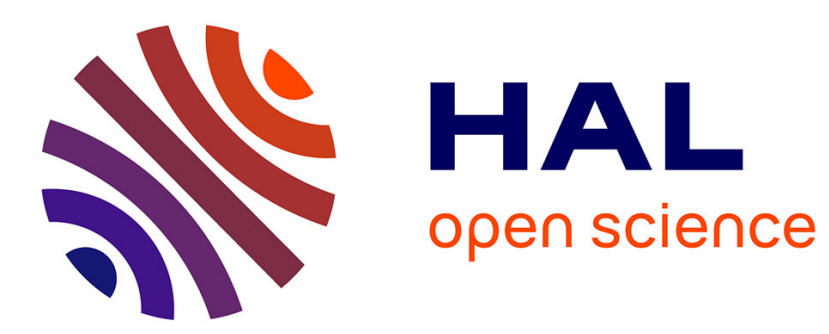

\title{
Les " sites doubles" drômois et ardéchois de la vallée du Rhône dans l'Antiquité
}

\author{
Jean-Claude Béal, Thierry Odiot
}

\section{To cite this version:}

Jean-Claude Béal, Thierry Odiot. Les " sites doubles " drômois et ardéchois de la vallée du Rhône dans l'Antiquité. Gallia - Archéologie de la France antique, 1999, Le Rhône romain, 56, pp.91-98. 10.3406/galia.1999.3246 . hal-01902784

\section{HAL Id: hal-01902784 \\ https://hal.science/hal-01902784}

Submitted on 16 Jan 2020

HAL is a multi-disciplinary open access archive for the deposit and dissemination of scientific research documents, whether they are published or not. The documents may come from teaching and research institutions in France or abroad, or from public or private research centers.
L'archive ouverte pluridisciplinaire HAL, est destinée au dépôt et à la diffusion de documents scientifiques de niveau recherche, publiés ou non, émanant des établissements d'enseignement et de recherche français ou étrangers, des laboratoires publics ou privés.

\section{(ㅇ)(1) $\$$}

Distributed under a Creative Commons Attribution - NonCommercial - NoDerivatives $\mid 4.0$ 


\title{
LES « SITES DOUBLES » DRÔMOIS ET ARDÉCHOIS DE LA VALLÉE DU RHÔNE DANS L'ANTIQUITÉ
}

\author{
Jean-Claude BÉAL et Thierry ODIOT
}

\begin{abstract}
Mots-clés. Sites doubles, vallée du Rhône, Drôme, Ardèche, Andance, Andancelle, Tain-l'Hermitage, Tournon, Valence, Cranges-lèsValence, Bourg-Saint-Andéol, Pierrelatte, traversée du fleuve (bacs, gués, ponts), commerce est-ouest.

Key-words. Duplex towns, the Rhône valley, Drôme, Ardèche, Andance, Andancette, Tain-l'Hermitage, Tournon, Valence, Granges-lèsValence, Bourg-Saint-Andéol, Pierrelaite, river crossing (pontoon bridges, fords, bridges), east-west trade.

Résumé. Une analyse critique des sources érudites, épigraphiques et archéologiques permet de reprendre le dossier des " sites doubles " de la vallée du Rhône. Les données concernant les sites d'Andance (07) et Andancette (26), Tain-l'Hermitage (26) et Tournon (07), Valence (26) et Granges-lès-Valence (07), Bourg-Saint-Andéol (07) et Pierrelatte (26) sont reprises et critiquées. Les " siles doubles "paraissent avoir été largement surévalués et pour beaucoup seraient de simples sites de passage d'une rive à l'autre. Cette relecture des sources permet de poser le problème de la traversée du fleuve (bacs, gués, ponts) durant l'Antiquité. Sont abordées aussi les notions de villes jumelles, de territoire et surtout de commerce est-ouest.
\end{abstract}

\begin{abstract}
Through a review of historical, epigraphical and archaeological sources, the case of the duplex towns in the Rhône valley can be taken up again. Facts concerning the following sites are being re-examined and discussed. Its seems that duplex lowns have been widely overemphasized and most of them must only have been setllements for river crossing. With this new reading of evidence, the question of the river crossing (pontoon bridges, fords, bridges) during Antiquity can be posed. Other notions being considered : twin towns, territory and east-west trade especially.
\end{abstract}

Dans la vallée du Rhône ${ }^{46}$, la notion de " sites doubles " ou de "villes-doublets " ${ }^{47}$ s'est imposée aux historiens et aux archéologues qui ont tenté de réfléchir

46. Par " vallée du Rhône ", nous entendons ici la section où lc fleuve forme la limite entre les actuels départements de l'Ardèche et de la Drôme. Pour une localisation générale des sites compris entre Tain et Bourg-Saint-Andécol, on se reportera à la fig. 34 , p. 85 .

47. L'expression est familière aux géographes : voir J. Beaujeu-Garnier, Géographie urbaine, Paris, éd. A. Colin, 1980, p. 75 (exemples de "villes bipartites " et de "villes jumelles"), et, pour la vallée du Rhônc, D. Faucher, 1968, p. 178 ; l'expression de « villes-doublets " serait due à A. Allix. En archćologie, le phénomène est signalé par excmple dans R. Chevallier, Les voies romaines, Paris, Librairie Armand Colin, 1972, p. 228 , note 3 . au réseau des villes et des agglomérations secondaires proches du fleuve. Décrivant la rive ardéchoise du Rhône, C.-A. Mazon considérait le fleuve non comme une séparation, mais comme un trait d'union entre les deux rives, d'où : " aux époques les plus reculées, [...] l'existence de ces villes doubles à cheval (sur le fleuve), villes sœurs que la politique a séparées. " ${ }^{48}$; de son côté, A. Blanc a évoqué les «villesjumelles : Andance-Andancette, Saint-Vallier-Sarras, Tain-

48. D) Francus (i. e. C.-A. Mazon), Voyage autour de Crussol, Privas, 1888 (rééd. 1977, Aubenas, Imp. Iicnhardt), p. 18; voir aussi P. Arnaud, 1966, p. 109-110. 


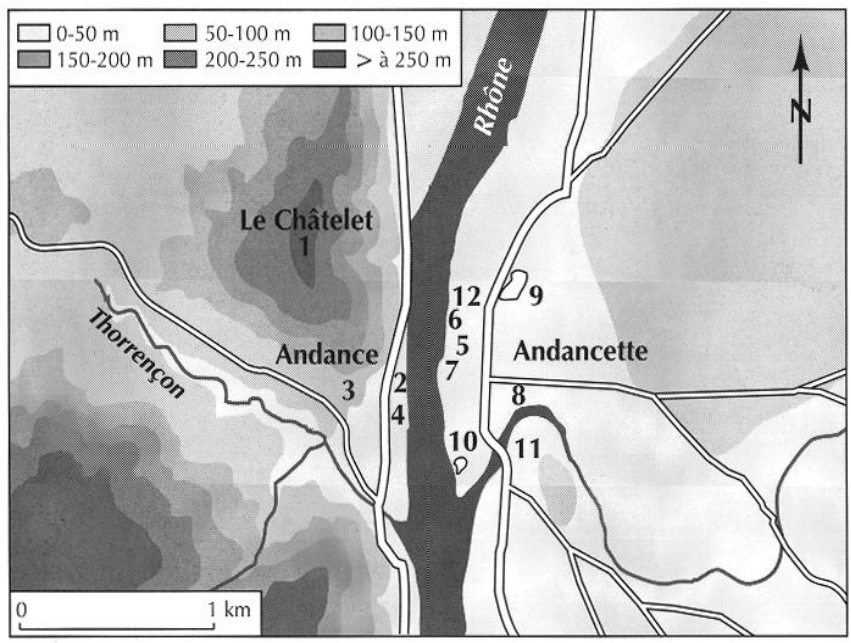

Fig. 35 - L'agglomération antique d'Andance/Andancette (dessin F. Vaireaux).

Andance: 1 , sanctuaire et hameau antique du Châtelel ; 2, tronçon de voie; 3, Les Rivoires, mobilier céramique et moellons, dallage d'une voie; 4, emplacement de l'agglomération antique où des "lignes régulières de bâliments séparées par une voie ou rue [se dirigent] du midi vers le nord, parallèlement au cours du Rhône" (Caillet, 1867, p. 43-44). Des élémients lapidaires en réemploi ont également été signalés; mais ils peuvent provenir du sanctuaire du Châtelet.

Andancette: 5, four de potier (?), dolia, amphores; 6, argile et briques plus ou moins cuites, slatuette de Mercure; 7, sur la berge du chemin de halage : céramique, tombes à incinération au débouché du pont actuel; 8 , "aqueduc ", aboulissant à un réservoir en face du cimelière; 9, nécropole à incinération; 10, nécropole à incinération, débris d'inscription; 11, stèle funéraire (CIL XII, 1796); 12, trésor monétaire préromain et molilier gallo-romain. L'emplacement du village actuel d'Andancette a également livré une autre inscription funéraire (perdue) et un trésor monétaire de Valérien (Ioriot, Rémy, $\left.1988, n^{\circ} 4, p .16\right)$.

Tournon, qui furent toujours reliées entre elles " ${ }^{49}$. M. Leglay, encore, a repris cette idée, et considéré comme " ville double "Vienne "à cheval sur le Rhône, comme Tain et Tournon, Valence et Crussol ou Soyons, Bances et Baix, Bourg-Saint-Andéol (Bergoiala) peut-être, Arles et Trinquetaille» (Le Glay, 1977, p. 312). C'est dire à quel point était admise l'idée d'une antique origine du phénomène. Cependant, un réexamen et une cartographie de la documentation archéologique invitent à nuancer beaucoup cette approche, comme le montre l'étude

49. (f. Blanc, 1975 , p. 34 ; l'auteur évoque l'existence d'unc aggiomération d'Ancône (Drôme) dont, en l'absence de vestiges antiques, on ne peut faire le symétrique de celle de Rochemaure sur la rive droite. de quatre sites principaux, à Andance, à Tainl'Hermitage, à Valence et à Bourg-Saint-Andéol ${ }^{50}$.

\section{ANDANCE ET ANDANCETTE}

$\mathrm{Au}$ nord de notre zone d'étude, Andance et Andancette apparaissent aux yeux des Modernes comme l'exemple même des sites doubles, jusque dans leurs noms dont l'un est la duplication de l'autre, avec emploi d'un diminutif plaçant Andancette, sur la rive gauche, dans la subordination d'Andance, sur la rive droite. Rappelons, du reste, que la création de la commune d'Andancette est tardive [1872], mais que le toponyme est attesté au XV's.

Pour l'Antiquité, les deux rives, qui appartenaient au territoire allobroge, ont livré les vestiges d'une bourgade sous les villages actuels (fig. 35).

Sur la rive droite, le bourg antique est installé, vers 130 m d'altitude, au pied de la colline du Châtelet, au sommet de laquelle, depuis l'époque augustéenne, se dresse un important sanctuaire régional; elle s'étend sur une étroite langue de terre, en pente vers l'est et le fleuve, et qui s'est formée à partir du cône de déjection du ruisseau du Thorrençon, à son confluent avec le Rhône ${ }^{51}$. La bourgade antique, de 4 ha au minimum, est traversée par la voie de la rive droite du Rhône, de Vienne vers Alba et Nîmes; une autre voie, d'orientation est-ouest, remonte la vallée du Thorrençon par la rive droite du ruisseau ${ }^{52}$.

50. À Sarras comme à Baix (Ardèchc), l'ćtat actuel de la documentation ne permet pas d'avancer l'hypothèse qu'une agglomération antique symétrique de celles de Saint-Vallier et du lieu-dit Bances à Saulce-surRhône (Drôme) ait cxisté.

51. Fouillé par I..-B. Morel au XIXes, le site cultucl a été réétudié par l'un d'entre nous (J.-C. B) en 1994. Un petit hameau antique s'est installé sur la colline, au sud du sanctuaire. Pour le bourg de la rive du flcuve, aux découvertes ancicmncs s'ajoutent quelques informations plus récentes. En attendant la publication (en cours de préparation), voir Blanc, 1975 , p. $76-78, \mathrm{n}^{\circ} 123$.

52. A. Blanc a supposé que la voie de la rive droite du Rhône était, ici comme ailleurs, doublée par un second itinéraire, qui contournait par l'ouest la colline du Châtelet et auquel on recourait " en période de hautes eaux" (Blanc, 1975, p. 30). Les témoins archéologiques de cette voie font encore défaut, $c t$ l'hypothèse d'A. Blanc repose sur l'idée que le niveau du Rhône était, dans l'Antiquité, au moins aussi haut qu'aujourd'hui, ce dont on peut douter : voir dans ce dossier la contribution de J.-P. Bravard et al., p. 13-32 ct de T. Odiot et C. Jung, p. 81-90. 
Sur la rive gauche, en vis-à-vis d'Andance, Andancette a été identifiee par certains érudits aux Figlinae mentionnées dans les itinéraires antiques comme étape sur la voie de Vienne en Arles (Sautel, 1957, p. 107, $\mathrm{n}^{\circ}$ 149). Toutefois, plusieurs auteurs ne sont pas d'accord avec cette identification, qui ferait du bourg de la rive gauche le noyau central de l'agglomération (Vallentin, 1880; Morel, 1928). La voie d'Agrippa passe à proximité de la bourgade actuelle, et de nombreux chemins, qu'il reste à dater, se dirigent depuis la plaine vers le sud du confluent ou vers l'emplacement de l'agglomération moderne. L'ensemble de ces itinéraires, qui drainent une grande partie des communications est-ouest de la plaine, semble traduire l'existence d'une intense circulation entre les deux rives. Le village actuel est implanté sur le cône de déjection du Bancel, légèrement au nord de son confluent avec le Rhône. Cet emplacement semble propice à l'installation d'une halte de batellerie (Ollier, 1958).

Toutefois, les vestiges, pour la plupart inventoriés sur près de 4 ha par L.-B. Morel, semblent moins denses que sur la rive ardéchoise ${ }^{53}$, même si les alluvions du Bancel et du Rhône peuvent avoir modifié la topographie du confluent et masqué d'éventuelles traces d'occupation. À comparer la documentation concernant ces deux quartiers, on a l'impression qu'une unique agglomération se développe sur les deux rives, d'autant qu'à cette hauteur, le fleuve est assez facilement franchissable par bac. Les quartiers d'habitation seraient plutôt sur la rive droite, au carrefour de deux voies terrestres et de la voie fluviale ; sur la rive gauche, se trouveraient plutôt les aménagements périphériques, nécropoles et four de potier peut-être, d'une bourgade pour laquelle le sanctuaire du Châtelet constitue un pôle d'attraction.

\section{TAIN ET TOURNON}

Sur la rive gauche du Rhône, Tain-l'Hermitage passe pour recouvrir la Tegna des itinéraires routiers antiques (fig. 36). L'agglomération, en territoire allobroge ${ }^{54}$, était desservie par la voie d'Agrippa de la rive gauche, dont on

53. Voir L.-B. Morel, 1928. Plus récemment, des fragments d'amphore Dr. 20 ont été découverts lors d'une construction (source : Carte archéologique du Service régional de l'archéologie de Rhône-Alpes, fig. 35, $\mathrm{n}^{\circ} 5$ ).

54. L'appartenance de Tain à la cité des Allobroges est certaine, la limite avec le territoire colonial de Valence pouvant être marquée ici

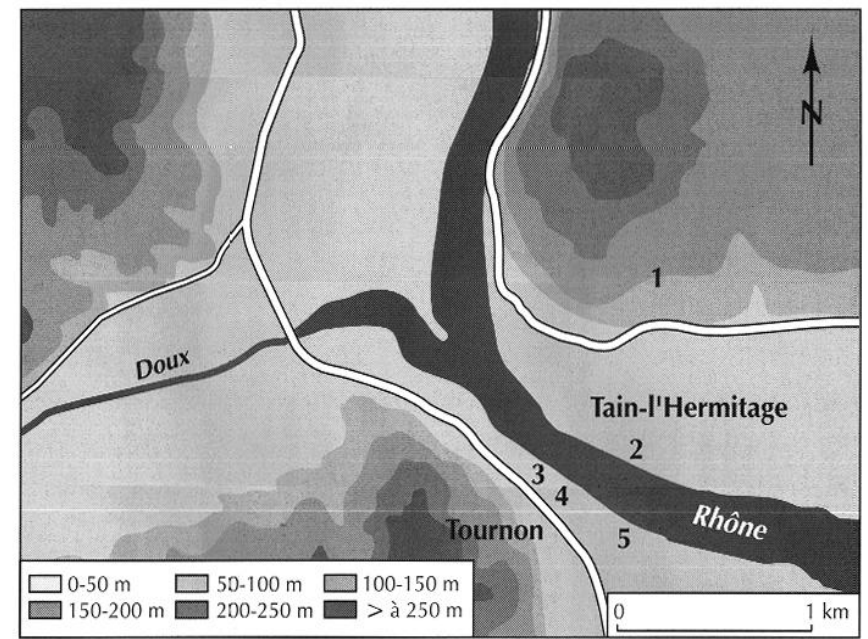

Fig. 36 - L'agglomération antique de Tain et Tournon (dessin F. Vaireaux).

Tain : 1, quartier de l'Hermitage ; 2, ilot Breysse-Michelon.

Tournon : 3, place A.Faure, vestiges d'un habitat ; 4, rue Centrale, débris de tuiles et d'amphores; 5, lycée de Jeunes Filles, deux tombes à incinération en amphores du II' $s$. de notre ère. Un ex-voto, offert par un sevir augustal, est signalé en réemploi dans la maison Ronzier, actuellement la sous-préfecture (CIL XII, 265I).

admet qu'elle arrivait à Tain par le nord, au pied du coteau de l'Hermitage. Plus près de la ville actuelle, la localisation des tombes isolées ou des nécropoles inciterait à proposer à la voie antique un tracé passant sur le coteau, légèrement plus haut que l'actuelle RN 7 .

Célèbre pour son autel taurobolique, la commune livre surtout des vestiges dans le secteur de l'Hermitage et sur les pentes (Sautel, 1957, p. 104, $\mathrm{n}^{\circ} 138$ ) ; mais, jusqu'à une date récente, aucune fouille n'avait permis d'étudier les éventuels vestiges antiques sous le centre urbain actuel.

Or, dernièrement, des sondages ont rencontré ${ }^{55}$, à proximité de l'église actuelle, des témoins d'une occupd-

par le confluent de l'Isère et du Rhône (Blanc, 1964, p. 23 ; Rémy, 1970, p. 213). Nous n'évoquerons pas ici les problèmes que pose le site de Saint-Estève (Blanc, 1975, p. 73-74, $\mathrm{n}^{\circ}$ 106), qui a pu être un port privé en rive droite du fleuve, ni l'inscription CII. XII, 1797 (dédicace des nautes du Rhône), déplacée plusieurs fois ( $c f$. infra, Christol, Fiches. p. 146-147).

55. Cf. F. Vaireaux, Tain-l'Hermitage, îlot Breysse-Michelon, Bilan scientifique 1993 de la région Rhône-Alpes, Lyon, 1994, p. 78-79; P. Réthoré, Tain-l'Hermitage, îlot Breysse-Michelon, Bilan scientifique 1994 de la région Rhône-Alpes, Lyon, 1995, p. 93. Nous tenons à remercier P. Réthoré pour son aide et pour la communication de ses documents personnels. 
tion de l'Âge du Bronze, et, après un hiatus, de la fin du $\mathrm{IV}^{\mathrm{c}} \mathrm{s}$. de notre ère ${ }^{56}$. En outre, ces traces d'occupation se situent dans une séquence stratigraphique où semblent se mêler les limons de débordement du Rhône et les apports détritiques de son affluent. Le centre de la ville moderne se serait donc développé à une date récente, sur un secteur de bras morts du Rhône, puisque les premières structures maçonnées n'apparaissent qu'à partir du XIV ${ }^{e}$ s. ; l'agglomération antique, quant à elle, se trouverait bien, pour l'essentiel, à l'est de la RN 7 ; hypothèse qu'il reste à confirmer par des sondages archéologiques.

Sur la rive droite du fleuve, Tournon, dont l'appartenance au territoire allobroge a été discutée (Blanc, 1982, p. $83-84, n^{\circ} 48$, contra Rémy, 1970, p. 207), occupe, au sud du confluent du Doux et du Rhône, un piton rocheux contre lequel le fleuve vient buter avant d'être repoussé vers l'est, formant un méandre que recouvrent en partie, entre $140 \mathrm{~m}$ et $120 \mathrm{~m}$ d'altitude, la basse ville et les quartiers récents. Le tracé de la voie de la rive droite n'est pas ici précisément connu ${ }^{57}$, et la rareté des vestiges antiques rencontrés n'y suggère pas, pour l'heure, une occupation dense (Blanc, 1975, p. 73, $\mathrm{n}^{\circ} 107$; Anonyme, 1984). Si une structure d'habitat est signalée, accolée au rocher devant le château, sa superficie et son organisation restent indéterminées. Des incinérations en amphores ont été trouvées un peu plus au sud et au bord du fleuve (fig. $36, \mathrm{n}^{\circ} 5$ ). Leur profondeur d'enfouissement montre qu'au voisinage immédiat d'autres structures peuvent exister et avoir échappé jusqu'à maintenant aux archéologues; mais ces tombes témoignent aussi qu'on est ici en dehors d'une zone d'habitat au II ${ }^{e}$ s. après J.-C.

56. Les opérations de sondages et de fouilles n'ont pas permis d'atteindre le substrat géologique. Plusieurs niveaux de limons sableux du Rhônc ont ćtć observés ; ils sont recoupés par une série de chenlaux interprétés comme des cours fossiles du Gréffieux, petit affluent du Rhône. C'est dans cette séquence stratigraphique qu'ont été retrouvées des céramiques du Bronze final. À un niveau supérieur, quelques céramiques du Haut-Empire attestent la présence d'une occupation dans le secteur. P. Réthoré pense qu'après une crue, qu'il faut situer entre le $\mathrm{I}^{\text {er }}$ et le II ${ }^{\mathrm{s}} \mathrm{s}$. de notre ère, le Gréffieux a changé de lit. Plus tard, il reçoit, dans son comblement, des traces d'une occupation de la fin du IV ${ }^{\mathrm{e}} \mathrm{s}$. Ces niveaux correspondent peut-être à des terres cultivées. La nécropole qui s'installe dans le secteur est datée du Haut Moyen Âge. Les premières traces de bâti sont datées du XIV $\mathrm{s}$.

57. A. Blanc considère que le " pont de César, construit sur le Doux à quatre kilomètres à l'ouest de Tournon, permettait de franchir en période de hautes eaux la rivière qu'on traversait à gué et plus directement lors des basses eaux » (Blanc, 1975, p. 30 et fig. 1).
$\mathrm{Au}$ contraire d'Andance et Andancette, il ne semble donc pas, en l'état actuel de la documentation, que l'on puisse évoquer ici, pour l'Antiquité, l'existence d'une agglomération des deux rives ou de deux agglomérations jumelles. L'existence même d'un faubourg en rive droite est incertaine.

\section{VALENCE ET GRANGES-LÈS-VALENCE}

La colonie romaine de Valence a été installée sur la rive gauche du fleuve (fig. 37), à l'endroit où le Rhône vient buter dans les terrasses anciennes et décrit une courbe vers l'est ${ }^{58}$, d'autant plus forte que les alluvions du ruisseau du Mialan ont créé un cône de déjection sur la rive droite et dans la plaine de Granges (Faucher, 1968, p. 93).

La ville est traversée par la voie de Lyon à Arles, et est aussi le point de convergence de plusieurs itinéraires (Sautel, 1957, p. 110-114; Barruol, 1975). Mais l'importance de la ville et du port de Valence tient aussi à la confluence de l'Isère et du Rhône, en l'état actuel à 6 km au nord de la ville ${ }^{59}$.

Du port antique de Valence, on ne sait à peu près rien. Il est localisé par A. Blanc en amont du pont présumé, en rive gauche et sur le territoire de l'actuelle commune de Bourg-lès-Valence (Blanc, 1964, p. 107; 1980, p. 9), où le vallon du Barberolle donnait un accès facile au fleuve depuis la ville haute. Les résultats des sondages menés quai de la Libération avant 1988 sont mal connus ${ }^{60}$; P. Valette a signalé un quai et de nombreux vestiges détruits lors de la construction d'immeubles dans ce quartier. En fait, les fouilles menées au quartier de la Pêcherie (Odiot et al., 1984) ont montré, dans une

58. Sur l'intérêt d'une position dans le coude externe du fleuve, voir Barruol, 1975 (qui la qualifie de " particulièrement favorable à l'installation d'un port fluvial»)

59. On rappellera cependant ici que l'hypothèse d'une rupture de charge à la hauteur du confluent de l'Isère et du Rhône repose sur une restitution imprudente d'un passage de l'Histoire d'Ammien Marcellin (XV, 11, 18) : voir J. Rougé, Compte rendu de Ammien Marcellin, Histoire, livres XIV-XVI, texte établi et traduit par E. Galletier (en collaboration avec J. Fontaine), Paris, 1968, Revue des Études latines, 1968 (1969), XLVI, p. 454.

60. Ces travaux sont cités dans A. Pelletier et al., 1988, p. 220 : « à Bourglès-Valence, sondages sur le port romain, ancien quai de la Verrerie, aujourd'hui de la Libération." 


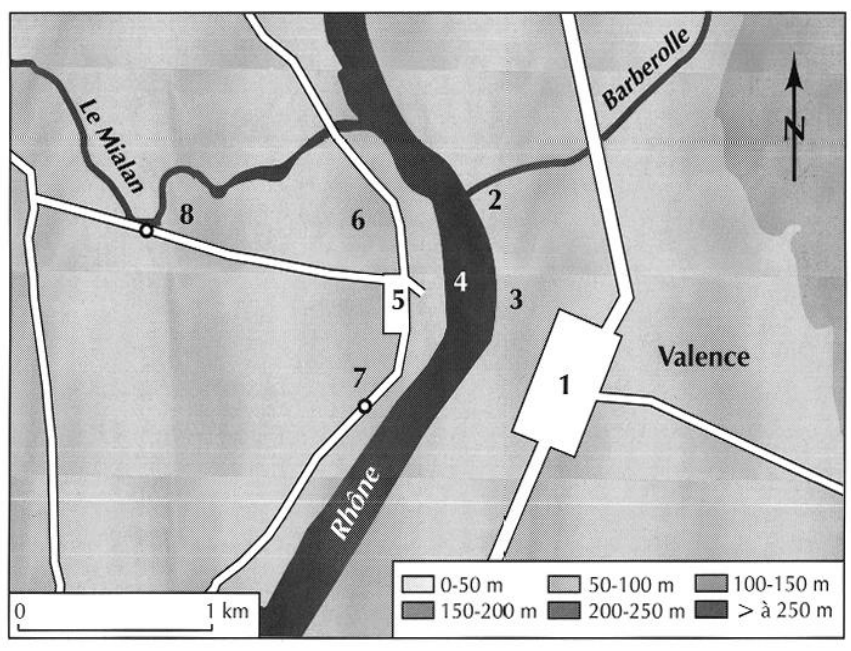

Fig. 37 - La colonie de Valence et le suburbium de Granges-lèsValence (dessin F. Vaireaux).

Valence : 1 , ville antique; 2 emplacement présumé du porl antique; 3, quartier des Pescheries.

Cranges: 4 , localisation approximative des pilotis et des restes de piles de maconnerie présumés antiques, fragments de mosä̈que; 5 , quartier des Nonniers, vestiges de constructions antiques; 6 , site du "Château Girodet ", construction et mobilier antiques, sépultures pour partie au moins médiévales; 7 , voie présumée de Cranges à Soyons : tombes et mobilier céramique antique; 8 , voie de la rive droite du Rhône, en coupe dans le cours du Mialan.

D'autres siles sont connus dans la plaine de Granges, au Pastouriaux, au Poulat, à Valenson, etc. Il paraît s'agir d'habitats isolés.

puissante séquence de limons de crues, que les vestiges médiévaux se situaient à plus de $2 \mathrm{~m}$ de profondeur; mais les éventuels niveaux antérieurs, plus profonds, n'ont pas été touchés par les sondages. C'est dire qu'ici comme à Tain, il faut prendre en compte la possibilité de recouvrement, sur plusieurs mètres d'épaisseur, par des limons masquant les vestiges antiques.

Sur la rive droite, censée appartenir au territoire colonial de Valence (Blanc, 1975, p. 30), la voie de Vienne vers $A l b a$ et Nîmes passe à moins de $2 \mathrm{~km}$ du fleuve. Selon certains historiens locaux, elle serait doublée par une voie de rocade, plus proche du fleuve. En tout cas, elle était reliée à la rive du Rhône par une voie rectiligne, d'orientation ouest-est, dont le dallage est apparu dans le lit du Mialan (Blanc, 1964, p. 104-405), et dont le tracé est repris à l'époque moderne ${ }^{61}$.

61. Cf. Blanc, 1975, p. 71. Selon cet auteur, le pont était « dans le prolongement du decumanus " principal de la ville (Blanc, 1975, p. 34).
Près du fleuve, quelques structures d'habitat mitoyennes de cet axe ont été reconnues au quartier des Nonniers et peuvent constituer un noyau de peuplement aggloméré, qui s'étendrait sur environ $100 \mathrm{~m}$ à l'ouest de la rue Pierre-Curie et $200 \mathrm{~m}$ au sud de la rue Henri-Dunant ${ }^{62}$. Mais, si dans ce quartier aujourd'hui totalement urbanisé et devenu inaccessible, on se fonde sur les témoignages de prospecteurs antérieurs à 1970, il ne semble pas que ce noyau forme un ensemble continu avec les autres points de découvertes de la plaine de Granges. Ainsi Mellier précise-t-il que c'est " par intervalle " que l'on rencontre ici des vestiges en prospection (Mellier, 1902-1903, p. 107). Des tombes sont signalées aussi bien au nord de cette zone qu'au sud, longeant la voie de rocade (Blanc, 1964, p. 85).

Cependant, à partir de ces quelques découvertes, $\mathrm{Y}$. Burnand ${ }^{63} \mathrm{a}$ avancé l'hypothèse que l'on pouvait avoir là un véritable quartier valentinois, à inclure dans le périmètre urbain de Valence, au contraire de la lecture de la ville faite par $\mathrm{A}$. Blanc, qui limite la ville antique à la rive gauche du fleuve.

Cette proposition est fondée principalement sur deux éléments, qui visaient à établir un parallèle avec le quartier viennois d'outre-Rhône à Saint-Romain-enGal, révélé à partir de 1968. Le premier concerne la richesse supposée d'un quartier " aux nombreux sols mosaiqués ", rappelant les découvertes de SaintRomain-en-Gal, ce que dément la réalité des faits constatés. En effet, seules deux mosaïques sont signalées sur cette rive : l'une trouvée en prospection concerne le site de la "Maison Girodet ", apparemment distinct du noyau de peuplement des Nonniers; l'autre est une " mosaique de cubes [...] déjà détruite par des travaux agricoles " découverte en 1960 (Lavagne, 1979, p. 136, $\left.\mathrm{n}^{\circ} 181\right)$.

Le second argument est celui de l'existence d'un pont assurant, comme à Vienne, la liaison entre les quartiers des deux rives.

L'existence de ce pont est débattue depuis longtemps. E. Mellier a d'abord insisté sur « l'indispensabilité (sic)

62. Voir la carte dressée par P. Valette en 1955 et reproduite dans P. Payen, 1977, à compléter par J.-P. Boucher, Informations archéologiques, circonscription de Rhône-Alpes, Gallia, 38, 2, 1980, p. 507 et J. Lasfargues, Bilan 1984, dans Ardèche-Archéologie, n² 2, juin 1985, p. 49. 63. Cf. Burnand, 1977, p. 304-305. Rappelons que M. Le Glay, de son côté, proposait de considérer Soyons, ou Crussol, comme ville double de Valence (Le Glay, 1977, p. 312). 
d'un pont sur le Rhône ", au vu de l'importance des vestiges attestés sur la rive droite, et du fait de la nécessité de mettre Valence en communication par route avec Alba (Mellier, 1902-1903, $144^{\mathrm{e}}$ livr., p. 107 et $145^{\mathrm{e}}$ livr., p. 183-198). Il a également mentionné l'existence, dès 1388, du toponyme de Pont Peri, qui peut rappeler un pont antérieur à celui, inachevé, du XIII ${ }^{c}$ s. A. Blanc, quant à lui, évoque deux découvertes archéologiques concernant, l'une et l'autre, le lit du fleuve près de son actuelle rive droite, dans le prolongement de la voie ouest-est. Il s'agit d'une part de pilotis de chêne, us encore au début du $\mathrm{XIX}^{\mathrm{c}} \mathrm{s}$., et qu'il rapporte à un pont, d'autre part de " restes de maçonnerie très importants en béton qui appartenaient aux piles du pont gallo-romain ", restes découverts dans les années soixante (Blanc, 1964, p. $104105 ; 1975$, p. 34 et $71 ; 1980$, p. 9 , note 6 ).

On notera cependant que les pilotis peuvent appartenir aussi bien à des appontements ou à un quai, qu'il faudrait dater. La découverte des blocs de maçonnerie reste, elle aussi, en l'état, difficile à interpréter, et ne garantit pas l'existence d'un pont. Au reste, on ne peut exclure que les vestiges décrits par $\mathrm{A}$. Blanc dans le cours actuel du fleuve appartiennent en fait à des structures d'appontement, voire au quartier d'habitat antique lui-même, car les variations latérales du cours du Rhône peuvent avoir gagné sur la rive antique. Dans cette perspective, la mosaïque signalée par $\mathrm{H}$. Lavagne, si elle était exactement positionnée sur le plan, fournirait un argument intéressant ( $c f$. supra et fig. $37, \mathrm{n}^{\circ} 4$ ).

Dès lors, si l'existence d'une liaison entre les deux rives du fleuve ne fait pas de doute ${ }^{64}$, la réalité matérielle d'un pont reste à établir, et, pour l'heure, on ne peut considérer le petit faubourg antique de la rive droite du fleuve autrement que comme un suburbium modeste, entouré de quelques villae et de nécropoles dans la plaine de Granges.

\section{BOURG-SAINT-ANDÉOL ET PIERRELATTE}

Bourg-Saint-Andéol, dont l'agglomération actuelle se trouve sur la rive droite du Rhône, constitue dans la tra-

64. Un point de passage a pu exister sur le Rhône à la hauteur du site de Valence dès l'époque protohistorique, voir Beeching et al., 1989, fig. 10 et p. 169, contra Barruol, 1975.

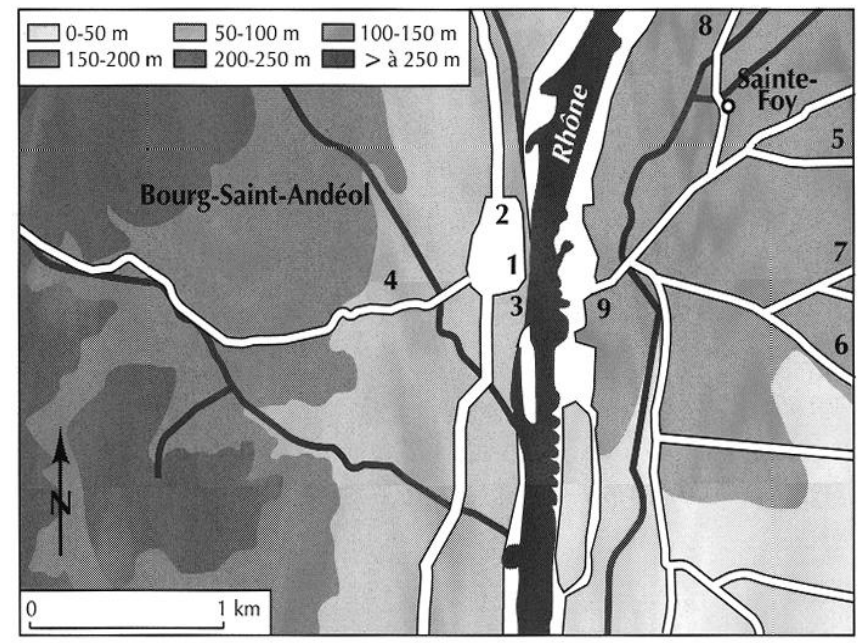

Fig. 38 - L'agglomération de Bourg-Saint-Andéol et Pierrelatte (dessin F. Vaireaux).

Bourg-Saint-Andéol : 1, église Saint-Polycarpe, constructions antiques; quartier Saint-Sauveur, construction et nécropole pour partie antique sans doute; 2, quartier Saint-Michel, pan de mur réputé antique ; 3, boulevard Edouard-Rambaud, mobilier antique, inscriptions votives; 4 , mithraeum au quartier de Tourne.

Pierrelatte: 5, site antique de Bourg, Pousse Bourg et Haute Bourgogne; 6, voie vers Saint-Paul-Trois-Châteaux; 7, voie vers Pierrelatte; 8, voie vers Donzère; 9, haut-fond rocheux et gué entre Pierrelatte et Bourg-Saint-Andéol.

dition historique régionale le cas le plus intéressant de doublet (fig. 38) : un port double y est attesté au IX ${ }^{e}$ s. de notre ère, une viguerie bourguésane de la rive gauche au tournant de l'an Mil (Lauxerois, 1983a, p. 52 et 116), et le récit de la passion d'Andéol, élaboré au IX ${ }^{\mathrm{c}} \mathrm{s}$., fait état d'un bourg de Bergoiate en rive gauche, auquel s'oppose en rive droite un site anonyme où le martyr est enterré, et qui deviendra Bourg-Saint-Andéol ${ }^{65}$.

Sur la rive droite, d'après la tradition locale, l'agglomération actuelle de Bourg-Saint-Andéol serait traversée par la voie de la rive droite du Rhône, venant de Viviers en direction de Saint-Just et de la rivière Ardèche. Son tracé serait repris dans le « Chemin Droit » et la Grande Rue actuels (Labrély, 1928, p. 74, plan h. t. ; Arnaud, 1966, p. 146 ; Lauxerois, 1983a, p. 113, fig. 5). De cette

65. Pour tout ce dossier, voir R. Lauxerois, 1983a, p. 51-52, 115-117 et 205-215. Cet auteur a bien montré (p. 116) que l'hypothèse du site double s'étayait ici de l'existence d'une « série d'exemples similaires de villes doubles depuis Vienne jusqu'à Arles " : nous avons vu ci-dessus ce qu'il en était. 
voie se détacherait ici un embranchement conduisant, par Gras et Valvignères, vers la capitale des Helviens : on y connait, en amont de Bourg, plusieurs bornes milliaires ${ }^{66}$.

L'existence d'une agglomération antique est ici assurée, même si les vestiges matériels sont peu nombreux. Près du fleuve, des restes de maçonneries antiques et une nécropole à inhumation en partie gallo-romaine ont été signalés (Blanc, 1975, p. 36, n 4 ; Lauxerois, 1983a, p. 112). L'un et l'autre signalent aussi un pan de mur encore visible au sommet du bourg, au quartier de SaintMichel, dont l'attribution à l'Antiquité gallo-romaine est plus douteuse. Du mithraeum, attribué à la fin du $\mathrm{II}^{\mathrm{c}} \mathrm{s}$. ou au début du III $s$. après J.-C. (Lauxerois, 1983a, p. 170-174) subsiste, en place et encore visible, le relief taurobolique, aux abords de la résurgence du Val de Tourne ${ }^{67}$. Enfin, parmi les vestiges mobiliers, on retiendra au moins une inscription funéraire ${ }^{68}$, et une dédicace aux Matres découverte sur le boulevard Rambaud en 1980, accompagnées de mobilier antique (Lasfargues, 1982, p. 394 ; Lauxerois, 1983a, p. 241 , n 2 ; 1983b, p. 196).

Sur la rive gauche, C. Boisse, reprenant la tradition du martyr d'Andéol, a cherché à localiser Bergoiate sur la commune dc Picrrclattc, aux licux-dits Lc Bourg, Poussc Bourg, Haute Bourgogne (Boisse, 1968, p. 18 et 164). Cependant, les prospections systématiques et l'étude de photo-carto-interprétation entreprises dans la plaine de Pierrelatte permettent désormais de replacer ce problème dans un contexte nouveau et plus précis. Elles montrent en effet qu'à la hauteur de Bourg-Saint-Andéol la densité de sites est particulièrement importante sur la commune

66. Voir en dernier R. Lauxerois, 1983a, $\mathrm{n}^{\text {os }} 46-47-48$, p. 267-268. L'appartcnancc du bourg antiquc dc la rivc droitc à la citć helvicnne est considérée comme une évidence; cependant, l'agglomération pourrait être incluse dans l'emprise du cadastre B d'Orange et de la préfecture dont il est le reflet (Chouquer, 1983, p. 279-283 et fig. 2).

67. R. Turcan considérait que le mithraeum de Bourg-Saint-Andéol était, à la différence des autres, installé "en pleine nature " : voir $\mathbf{R}$. Turcan, Les religions de l'Asie dans la vallée du Rhône, Leiden, 1972, p. 44. L'état actuel de la documentation montre, nous semble-t-il, que, s'il est en marge d'une agglomération, il est bien installé, comme les autres, en habitat aggloméré.

68. CIL XII, 2707 ; Lauxerois, 1983a, p. $277, \mathrm{n}^{\circ} 68$. En revanche, selon R. Lauxerois, le sarcophage de Tiberius Julius Valerianus, trouvé à Bourg-Saint-Andéol et réputé avoir été réutilisé pour renfermer les restes de saint Andéol, pourrait aussi bien y avoir été apporté après la fin de l'Antiquité (Lauxerois, 1983a, p. 220-221). de Pierrelatte ( $c f$.Jung et Odiot, supra, p. 86). Tout semble indiquer que l'attraction économique de Bourg-SaintAndéol et la facilité de la traversée ont attiré de nombreux habitats, mais les prospections ne permettent pas de distinguer dans la vingtaine de sites repérés vis-à-vis de Bourg un ensemble de vestiges livrant un mobilier distinct de celui des villae environnantes et attribuable à une agglomération. En revanche, l'existence d'un appontement y est probable, ne serait-ce que pour répondre aux besoins d'exportation de la cité des Tricastins.

Ces sites s'organisent le long de plusieurs voies. Un axe majeur permet de rejoindre, en utilisant le dixième decumanus du cadastre B d'Orange, la voie d'Agrippa et Augusta Tricastinorum; une autre voie se dirige vers Pierrelatte ${ }^{69}$ et la vallée de la Berre ; une troisième, longeant le fleuve, permet de traverser la Berre et d'accéder à Donzère, puis au-delà du défilé à Montélimar, où elle rejoint la voie d'Agrippa.

L'ensemble de ces itinéraires converge vers un hautfond rocheux, situé immédiatement au sud du pont actuel de Bourg-Saint-Andéol, qui permet un passage à gué, relativement aisé en période sèche.

Ainsi, la voirie de la plaine tricastine est-elle largement influencée par les relations est-ouest, alors même quc l'on nc pcut disccrncr, à proximitć du Rhônc, d'habitat groupé qui, à l'époque gallo-romaine, répondrait à Bourg-Saint-Andéol ( $c f$. Jung et Odiot, supra, p. 86). Le même phénomène se reproduit plus au sud, où un autre haut-fond fait face à l'oppidum de Saint-Étienne-de-Dion. Ici encore, une grande voie traverse en diagonale la plaine de Pierrelatte depuis la vallée de la Berre pour rejoindre ce passage privilégié, sans pour autant qu'on identifie en face de l'oppidum un bourg jumeau de rive gauche.

Ainsi, parmi les sites réputés "doubles " que nous venons d'étudier, il n'y a d'abord pas de villes jumelles antiques comparables aux couples que formeront, plus tard, Tain et Tournon, Viviers et Châteauneuf-du-Rhône par exemple. Plus généralement d'ailleurs, les cas certains d'agglomérations jumelles semblent bien rares

69. La densité des constructions du centre urbain de Pierrelatte et l'absence de fouilles ne permettent pas de donner un statut aux vestiges rencontrés dans les années soixante à proximité de la chapelle des Pénitents (ramassages Del Pappa, collection particulière visible au musée municipal de Pierrelatte). Les niveaux antiques ont livré les traces d'une occupation durant tout l'Empire. Sur le problème de Pierrelatte, voir T. Odiot, 1994. 
dans la Gaule antique : si Ratiatum-Rézé et CondevincumNantes se répondent à l'embouchure de la Loire, il y a $6 \mathrm{~km}$ et plusieurs bras de Loire entre le port des Pictons et celui des Namnètes (Provost, 1993, p. 139-140, note 161). On connaît assez bien Ugernum-Beaucaire, mais notre documentation est infiniment plus pauvre pour situer Tarusco-Tarascon qui paraît son symétrique sur la rive gauche du Rhône. C'est que, dans le cadre des cités défini par Rome, le fleuve est un axe économique, comme les routes qui le doublent, mais il n'est pas, comme il l'a peut-être été auparavant et le sera par la suite, le lieu privilégié d'un affrontement pour le contrôle du territoire ${ }^{70}$ et pour la levée d'octroi et de péage au profit d'autorités ou de communautés locales ${ }^{71}$.

On ne reconnaît pas davantage, parmi ces " sites doubles ", de véritables habitats agglomérés à cheval sur le fleuve, comme à Vienne ou en Arles, ou comme on croit le distinguer à Seveux-Savoyeux par exemple, de part et d'autre de la Saône ${ }^{72}$. Plusieurs sont en fait constitués d'une agglomération que, sur la rive d'en face, complète un modeste " faubourg-relais " ${ }^{73}$, sur la rive gauche à Andancette dans la dépendance d'Andance, sur la rive droite à Granges en face de Valence.

70. Ainsi les évêques de Viviers sont-ils seigneurs de Châteauneuf au XIII' ${ }^{\mathrm{s}}$., tandis que la seigneurie de Tain est sous le contrôle de la famille de Tournon (Brun-Durand, 1891, p. 78 et 383). Voir supra le cas de Bourg-Saint-Andéol après l'Antiquité.

71. Sur la levée de taxes locales, routières et fluviales, en Gaule à l'époque républicaine, et peut-être jusque sous Tibère, voir S. J. De I.aet, Portorium, Étude sur l'organisation douanière chez les Romains, surtout à l'époque du Haut-Fmpire, Bruges, 1949, p. 77-84. Par la suite, les taxes locales semblent avoir disparu et seules ont été levées les taxes au profit de l'état romain, à l'entrée en Gaule (en Arles par exemple), ou dans de grands centres de regroupement de marchandises (Lyon par exemple). Sur l'importance des points de contrôle aux époques suivantes, "occasion d'une levée de péage et de taxes divcrscs ", ct originc dc quarticr pćri-urbain ou d'agglomćration, voir M. Fixot, in : Duby G. (éd.), Histoire de la Fance urbaine, I, Paris, 1980, p. 534-535, ou plus particulièrement pour la vallée du Rhône, A. Allix, Le trafic en Dauphiné à la fin du Moyen Âge, Revue de Géographie alpine, 11, 1923, p. 420 (carte des péages sur le Rhône).
L'existence de ces faubourgs montre que l'importance du fleuve n'est pas telle, ici, que les points de débarquement doivent engendrer des structures très développées sur les deux rives. Au reste, les témoignages épigraphiques concernant la vie du fleuve se réduisent à bien peu de choses dans toute la zone étudiée. Ces faubourgs témoignent en revanche de l'importance des relations transversales entre les deux rives du fleuve, qu'à Andancette, à Granges, ou à Pierrelatte confirme la restitution d'un réseau routier antique en éventail à partir du fleuve. La même observation vaudrait du reste pour La Motte, commune de Portes-lès-Valence (Drôme), à la hauteur de l'agglomération antique de Soyons (Ardèche).

En outre, dans quelques cas (Andancette, Granges, et peut-être Tournon), l'existence d'une nécropole d'outre-fleuve ${ }^{74}$ suggère que le Rhône n'ait pas été perçu comme une limite au territoire commun du bourg principal, sans que, faute d'une documentation, épigraphique notamment, suffisamment précise et abondante, on puisse dire dans quelle mesure cette perception était transcrite en terme de découpage administratif du territoire ${ }^{75}$.

72. Voir S. Labre, Sevcux (IIaute-Saône), in : Mangin M., Jacquet B., Jacob J.-P. (éds), Les agglomérations secondaires en Franche-Comté romaine, Annales littéraires de l'université de Besançon, $n^{\circ} 337$, Paris, Les Belles Lettres, 1986, p. 91 : la parenté des toponymes Seveux et Savoyeux et "l'identité morphologique » du réseau sur les deux rives amène l'auteur à supposer qu'on ait ici « une seule et même agglomération ».

73. L'expression est aussi de D. Faucher, 1968, p. 178.

74. Ce pourrait être encore le cas à La Motte, en face de Soyons, où des tombes ont été reconnues (Sautel, 1957, p. 97, n $108^{i i s}$ ).

75. La qucstion sc posc ccpcndant d'abord au nivcau dc la citć : si l'agglomération d'Andance et son faubourg d'Andancette sont dans la cité des Allobroges, on ne sait trop par exemple, nous l'avons vu, si Tournon esı dans la même cité que le bourg de Tain. Ft le problème se pose aussi sans doute au niveau des subdivisions du territoire des cités. 